\title{
A Prototyped Ad Hoc Routing Protocol for Efficiency Enhancement for Routing in MANET
}

\author{
Pushpendra Dwivedi \\ Pranveer Singh Institute of Technology \\ Kanpur, U.P. (208020) \\ U.P.T.U., Lucknow
}

\author{
Saurabh Gupta \\ Pranveer Singh Institute of Technology \\ Kanpur, U.P. (208020) \\ U.P.T.U., Lucknow
}

\begin{abstract}
Discussing about An ad-hoc network in today's world is like, it is an emerging technology of wireless nodes forming a network that is infrastructure less. So it can be said that without any established infrastructure. There are a lot of routing protocols for mobile ad hoc networks to have efficient routing. In this paper there are two types of networks; one is overlay networks and the other is local ad-hoc networks. Overlay ad-hoc networks is having better results than local ad-hoc networks. At last this paper compares the performance of some of them. The main focus is to enhance the efficiency of routing protocols in ad-hoc networks. On the basis of some parameters the performance of existing routing protocols is also been tested. The efficiency check is done by using ns2 simulation.
\end{abstract}

\section{Keywords}

MANET, routing protocol, network simulation, infrastructure, overlay.

\section{INTRODUCTION}

A Delay-Tolerant Networks are overlay networks that operates on top of different local networks. These local networks also include the internet. DTNs allow local networks with differentdifferent delay properties to interoperate by providing different mechanisms to translate between their network parameters. So the underlying protocols for these local networks may differ considerably. The DTN's are connected to each other because of their flexibility [1] [2].

If internet is taken into consideration, then it can be said that it widely operates on one protocol that is TCP/IP, thus ensures a standard set of protocols. Worldwide a lot of devices are operating on internet. To provide end to end services, these type of protocols are responsible. Now the point to be discussed is the basic working of the protocols. The basic working is not affected by the variation in link-layer technology. These protocols are very much characterized by routes with low error rates, low delays and continuous connectivity over the time. However, these protocols may not be applicable to all kinds of networks, particularly in those that are under the constraints of high delays and losses.

When interoperability is taken into consideration, then delay tolerant networks lay important role. Because they support interoperability of one network with the other with the accommodation of long disruptions and delays also with the translation between the communication protocols of those networks. In today's scenario the wireless communication devices are used with their two basic requirements the mobility and limited power and DTNs support the both. The main use of delay tolerant networks is interplanetary. Because in these types of applications the speed of light seems slow, this phrase is said because they have a lot of delay with them, So DTNs have to be used in them. So nowadays DTNs have got a lot of application areas, where disruption-tolerance is the greatest need. The potential Earth applications span a broad range of commercial, scientific, military, and public-service applications.

\section{EPIDEMIC ROUTING}

Epidemic routing approach is being proposed for sparse mobile network. Epidemic routing working is usually based on the "Store-Carry-Forward" paradigm. In epidemic routing, randomly pair wise exchange of message is done among scattered mobile nodes to ensure message delivery. As store and carry forwarding routing approach usually works on the basis of space buffers. When any source node wants to send the information to the destination node then, firstly the message information is being saved to the buffer space from source node and then forwarded to the destination node. Hence when the traffic load is low, epidemic routing gains minimum delivery delay and have increased use of resources like buffer space, and bandwidth. In Delay tolerant network epidemic routing is being widely used as it provides shortest routing delays. So it can be said that in epidemic routing, the data has been replicated along different paths and the delay in delivering a data packet is hence the time to move a data packet along opportunistic path in shortest time.

\section{OVERLAY NETWORK}

In any nodal network, an overlay network exists "on top" of the infrastructure or it can be defined as the network which exists imaginary in any of the real node based network. It provides basic transport medium for telephony and other multimedia application and that multimedia application can be voice, video or data. Hence overlay provides diverse array of networks and new network technologies that exists "on top" of the infrastructure network. Hence main goal of any overlay network can be stated as:

1) It exists on top of an infrastructure network.

2) One or more application can be provided by infrastructure.

3) Forwarding, managing and handling of application data in various ways, this responsibility or service is also taken by an overlay network to provide the better service to the basic internet.

4) Third party users or customers/end users can operate such network in an organized and coherent manner.

Hence the concept Overlay network in delay tolerant network can be explained as when in any mobile Ad-hoc network, there exists an overlay network on the top of network then it can store and forward information or any request and helps in finding out the destination node early by searching and storing the information in any wireless or mobile Ad-hoc network. 


\section{GRAPHS AND RESULT}

When there is any simulation is done on ad-hoc networks, then it will be on the basis of some MANET application. Some applications are purely general purpose but some are of specific purpose. The report reviews the pure general-purpose MANETs as well as other specified MANETs. The application scenarios of pure general purpose ad-hoc networks are Battlefield or disaster-recovery networks. These are not truly achievable.

Multi-hop ad hoc network is formed with mesh networks built upon a mix of fixed and mobile nodes that are interconnected via wireless links. A mesh network is having a hierarchy in the network by adding mesh routers that communicate wirelessly to construct a wireless backbone. These mesh routers works like dedicated nodes.

Law enforcement agencies and city governments uses Wireless mesh networks. These type of networks are like the natural solutions to them. Recently, many mesh networks are implemented to provide public safety applications. The applications of delay tolerant networks are wildlife monitoring and internet connectivity to developing areas. There can be two types of applications of MANETs for low traffic and high traffic. Not all applications need high Packet delivery ratio. Some application examples are, like those who provide Internet connectivity to rural and developing areas. For driver assistance and car safety, we use VANETs. They have ad hoc communications for performing the task efficiently. This includes the data from the roadside and from other cars. The main research in VANET supports drivers with the information of obstacles on the road, mainly due to line-ofsight limitations and large processing delays.

\section{When the traffic is low}

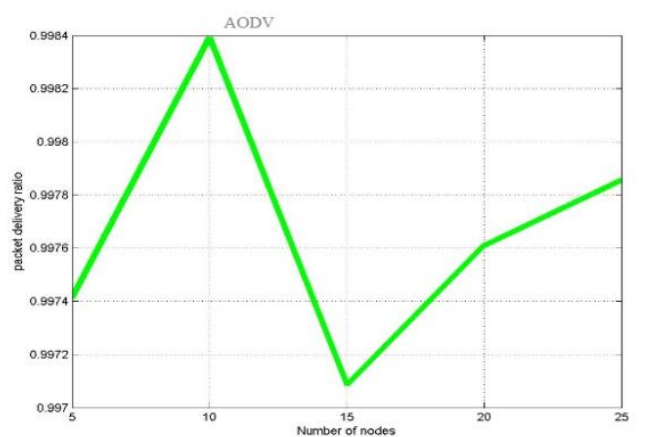

Fig 1: Packet Delivery Ratio v/s no. of node for AODV

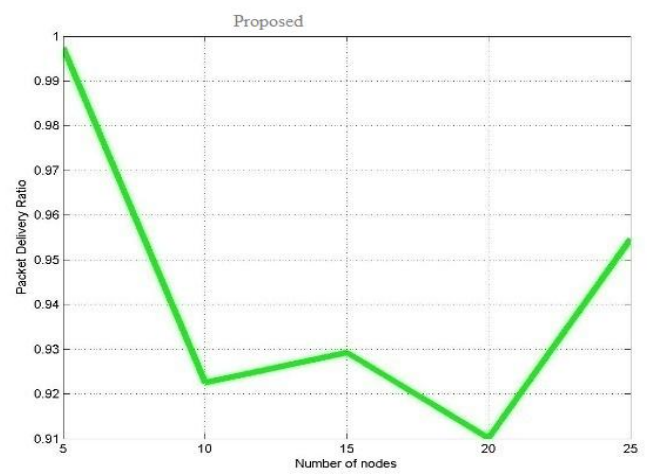

Fig 2: Packet Delivery Ratio v/s no. of node for Prototyped

The graph shows that in low traffic applications when the no. of nodes are increased then the packet delivery ration sharply decreased in the Prototyped algorithm. But in AODV it decreases and increases in zigzag motion. After a threshold of no. of nodes, the packet delivery ration starts increasing in prototyped algorithm. So at low traffic, when the no. of nodes are more than a threshold value, then the prototyped algorithm can have better packet delivery ration than AODV. As it is clear to see when the traffic is very low $(<10)$, Packet Delivery ratio of the Table Driven Protocol is better than that of prototyped.

\section{When the traffic is high}

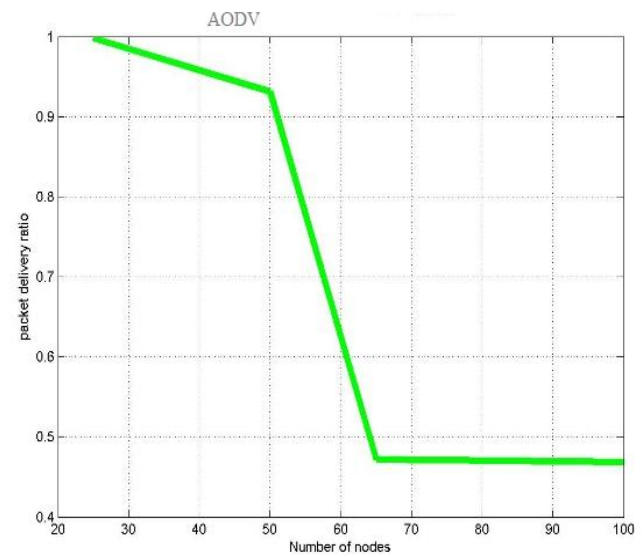

Fig 3: Packet Delivery Ratio v/s number of node for AODV

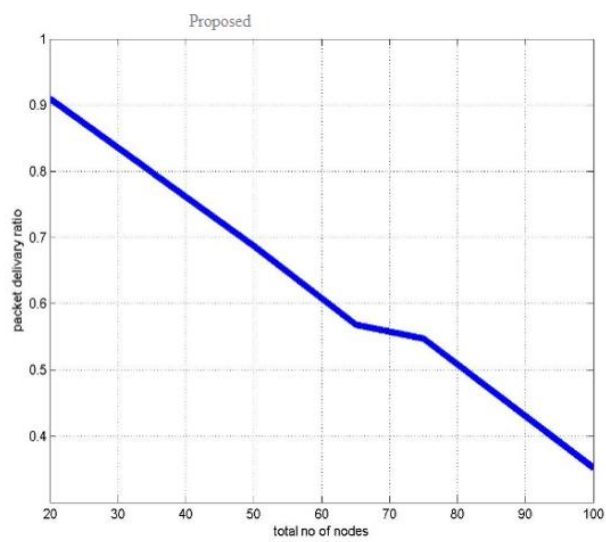

Fig 4: Packet Delivery Ratio v/s number of node for Prototyped

When the high traffic is considered then while increasing the no. of nodes, a sharp decrease in packet delivery ration is seen in AODV curve. But in prototyped algorithm curve shows a very slow decrease. So after increasing no. nodes at high traffic, we will have very less packet delivery ratio in AODV. So AODV will have lesser packet delivery than prototyped algorithm at high traffic also.

\section{CONCLUSION}

These simulations are done on ad-hoc networks, it is on the basis of some MANET application areas. That's why the simulation is done in two forms: one is at low traffic and the other is high traffic areas. The simulation results shown in this paper can conclude that the prototyped algorithm uses lesser bandwidth, when compared with the AODV routing protocol. In AODV the more traffic it has, less efficient it is. The comparison is done on the basis of increase and decrease in packet delivery ration. When the packet delivery ratio of any protocol is less then it means it will have less throughput and it 
is less efficient. We have examined packet loss for AODV and prototyped algorithm and from our simulation this can be concluded that in AODV the packet loss is mainly due to mobility, however in algorithm the packet loss is mainly due to congestion, but result of overlay is much better than AODV in throughput and performance. The effect of error messages is increased by sending all the messages to the sink. The future scope is to check the efficiency of other protocols in the hybrid situation.

\section{REFERENCES}

[1] Cerf, S. Burleigh, A. Hooke, L. Torgerson, R. Durst, K. Scott, K. Fall, H. Weiss, Delay-Tolerant Network Architecture, DTN Research Group Internet Draft, Draft 2, <draft_irtf_dtnrg_arch_02>, March 2003.

[2] Kevin Fall, A Delay-Tolerant Network Architecture for Challenged Internets, Intel Research Berkeley, Technical Report IRB-TR-03-003.

[3] S. Burleigh, V. Cerf, R. Durst, K. Fall, A. Hooke, K. Scott, L. Torgerson, H. Weiss, BundleLayer Protocol Specification, $\quad \mathrm{V} \quad 0.4, \quad 9 / 6 / 2002$, http://www.dtnrg.org/specs/blps 0.4.pdf.

[4] Scott Burleigh, Vint Cerf, Bob Durst, Adrian Hooke, Keith Scott, Eric Travis, Howard Weiss, The Interplanetary Internet: Status and Plans, DARPA NextGeneration Internet. (NGI) Network, (http://www.ngisupernet.org/NGI-PI-2001/Cerf.pdf), January 2002.
[5] Per Johansson, Tony Larsson, Nicklas Hedman, Bartosz Mielczarek, and Mikael Degermark. Scenario-Based Performance Analysis of Routing Protocols for Mobile Ad-Hoc Networks. In ACM/IEEE International Conference on Mobile Computing and Networking (MOBICOM), pages 195-206, 1999.

[6] David B. Johnson and David A. Maltz. Mobile Computing, chapter Dynamic Source Routing in Ad Hoc Wireless Networks, pages 153-181. Kluwer Academic Publishers, 1996.

[7] J. M. Kahn, R. H. Katz, and K. S. J. Pister. Next Century Challenges: Mobile Networking for Smart Dust. In Proceedings of the Fifth Annual ACM/IEEE International Conference on Mobile Computing and Networking,pages 271-278, 1999.

[8] P. Krishna, M. Chatterjee, N. H. Vaidya, and D. K Pradhan. A Cluster-Based Approach for Routing in Ad Hoc Networks. In USENIX Symposium on Location Independent and Mobile Computing, April 1995.

[9] S.B. Lee, A. Gahng-Seop, X. Zhang, and A.T. Campbell. Supporting Quality of Service in Mobile Ad Hoc Networks. In 8th IFIP International Conference on High Performance Networking (Network 2000),May 2000.

[10] Steve McCanne, Sally Floyd, and Kevin Fall. ns - LBNL Network Simulator. See http://www-nrg.ee.lbl.gov/ns/, 1996. 